\title{
Simulated Annealing Optimization Method on Decentralized Fuzzy Controller of Large Scale Power Systems
}

\author{
Mahmoud Najafi
}

\begin{abstract}
The centralized control of large scale systems is usually infeasible due to their high dimensionality, nonlinearity, coupling, time delay and etc. In order to overcome the mentioned difficulties, this paper presents an application of decentralized fuzzy logic controller (FLC) which is optimum by Simulated Annealing (SA) algorithm on two interconnected power area for the load frequency control as a case study of large scale systems. The simulation study is undertaken, based on MATLAB / SIMULINK to demonstrate and confirm the effectiveness of proposed method.
\end{abstract}

Index Terms-Fuzzy logic control; simulated annealing optimization method, large scale systems; load frequency control.

\section{INTRODUCTION}

For large scale electrical power systems that normally consist of interconnected control areas or regions representing coherent groups of generators, load frequency control (LFC) is very important in power system operation and control for supplying sufficient and reliable electric power with good quality. In cases of area load changes and abnormal conditions, such as outages of generation and varying system parameters, mismatches in frequency and scheduled tie-line power flows between areas can be caused. These mismatches are corrected by controlling the frequency, which is defined as the regulation of the power output of generators within a prescribed area [1]. The objective of the LFC is to satisfy the following requirements [2,3]: (i) Zero steady state errors in tie-line exchanges and frequency deviations. (ii) Optimal transient behaviors. (iii) In steady state, the power generation levels should satisfy the optimal dispatch conditions. In the past, several control designs of LFC in interconnected power systems has been studied. The conventional control strategy for the LFC problem is to take the integral of the control error as the control signal. An integral (I) controller provides zero steady state frequency deviation but it exhibits poor dynamic performance.

Over the past decades, many control strategies for load frequency control of power systems such as linear feedback [4], optimal control [5] and variable structure control [6,7] have been proposed in order to improve the transient response. Adaptive controllers with self adjusting gain settling have also been proposed for the LFC problem [8].

Manuscript received June 12, 2012; revised July 12, 2012.

Mahmoud Najafi was at the Control and Instrument Department of National Iranian Oil Company (IOOC ) and now is the ICT manager at IOOC (e-mail: mnajafi@iooc.co.ir )
Artificial neural networks have been successfully applied to the LFC problem with rather promising results $[9,10]$. Moreover, fuzzy logic control techniques for the LFC problem are mostly based on fuzzy gain scheduling of proportional integral (PI) controller parameters [11-13]. Recently, applications of fuzzy logic theory to the engineering issues have drawn tremendous attention from researchers [14]. The FLC has a number of distinguished advantages over the conventional controllers. It is not so sensitive to the variation of system structure, parameters and operation points and can be easily implemented in a large scale nonlinear system. Furthermore, the fuzzy logic controller is a sophisticated technique that is easy to design and implement.

The contribution of this paper is to propose a new approach based on the SA for optimal design of a fuzzy logic load frequency controller in a two-area interconnected power system. This proposed approach, called the SA algorithm, is used to obtain optimal or nearly optimal solutions very quickly; moreover, the efficiency of the proposed algorithm will be demonstrated in this paper. The paper is organized as follows: Section II, a comprehensive mathematical model of a load frequency control problem for a two area interconnected power system is presented. The proposed FLC is described in Section III. An application of the proposed SA algorithm to optimize fuzzy logic load frequency controller are presented in Section IV. The simulation results in a two-area interconnected power system are offered in Section V. Finally, the conclusion is provided in Section VI.

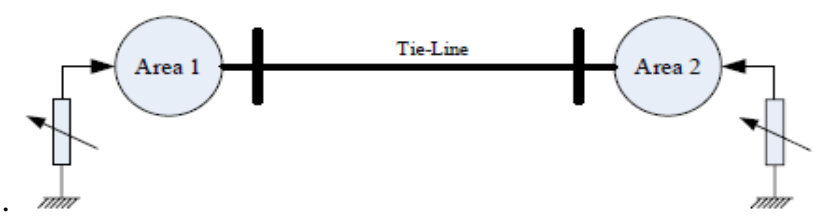

Fig. 1. Two-area interconnected power system.

\section{PROBLEM FORMULATION}

An interconnected power system is divided into control areas connected by a tie line. In each control area, all generators are supposed to constitute a coherent group. A two-area interconnected power system shown in Fig.1 is used to explain the motivation of the proposed method. It is assumed that large loads with sudden changes, such as large steel mills, arc furnace factories, magnetic levitation transporters and testing plants for nuclear fusions etc, have been placed in both areas. The frequency deviation in both areas severely affect the production quality of frequency 
sensitive industries such as the petrochemical industry, pulp and paper industry, semiconductor industry, etc.

Furthermore, the lifetime of machine apparatuses on the load side will be reduced.

The tie-line power flow and frequency of the area are affected by the load changes. Therefore, it can be Considered that each area needs its system frequency to be controlled. Large scale power systems can be represented using Differential Algebraic Equations .The differential and algebraic equations represent the generator dynamics and excitation and speed governor system equations respectively are given by:

$$
\left\{\begin{array}{l}
\frac{\mathrm{d} \delta_{i}}{\mathrm{dt}}=\omega_{\mathrm{i}}-\omega_{\mathrm{s}} \\
\frac{2 \mathrm{H}_{\mathrm{i}}}{\omega_{\mathrm{s}}} \frac{\mathrm{d} \omega_{\mathrm{i}}}{\mathrm{dt}}=\mathrm{T}_{\mathrm{mi}}-\mathrm{T}_{\mathrm{ei}}-\mathrm{K}_{\mathrm{di}} \Delta \omega_{\mathrm{i}} \\
\mathrm{T}_{\mathrm{doi}}^{\prime} \frac{\mathrm{dE}_{\mathrm{qi}}^{\prime}}{\mathrm{dt}}=-\mathrm{E}_{\mathrm{qi}}^{\prime}-\left(\mathrm{X}_{\mathrm{di}}-\mathrm{X}_{\mathrm{di}}^{\prime}\right) \mathrm{I}_{\mathrm{di}}+\mathrm{E}_{\mathrm{fdi}}
\end{array}\right.
$$

And

$$
\begin{aligned}
& \dot{\mathrm{E}}_{\mathrm{fd}_{\mathrm{i}}}=-\frac{\Delta \mathrm{E}_{\mathrm{fd}_{\mathrm{i}}}}{\mathrm{T}_{\mathrm{Ai}}}+\frac{\mathrm{K}_{\mathrm{A}_{\mathrm{i}}}}{\mathrm{T}_{\mathrm{Ai}}} \Delta \mathrm{V}_{\mathrm{i}_{\mathrm{i}}}+\frac{\mathrm{K}_{\mathrm{A}_{\mathrm{i}}}}{\mathrm{T}_{\mathrm{Ai}}} \mathrm{U}_{\mathrm{f}_{\mathrm{i}}}(\mathrm{AVR}) \\
& \dot{\mathrm{P}}_{\mathrm{i}}=-\frac{\mathrm{P}_{\mathrm{i}}}{\mathrm{T}_{\mathrm{Gi}}}+\frac{\mathrm{K}_{\mathrm{g}_{\mathrm{i}}}}{\mathrm{T}_{\mathrm{Gi}}} \frac{\Delta \omega_{\mathrm{i}}}{\mathrm{W}_{0}}+\frac{\mathrm{P}_{\mathrm{mo}_{\mathrm{i}}}}{\mathrm{T}_{\mathrm{Gi}}}+\frac{\mathrm{K}_{\mathrm{Gi}}}{\mathrm{T}_{\mathrm{Gi}}} \mathrm{U}_{\mathrm{Gi}}(\mathrm{GOV})
\end{aligned}
$$

where $\mathrm{x}_{\mathrm{di}}^{\prime}$ is the transient reactance and $\delta_{i}$ is the power angle of the $i$-th generator in $\mathrm{rad}, \omega_{i}$ is the rotating speed of the $\mathrm{i}$-th generator in $\mathrm{rad} / \mathrm{s}, \mathrm{H}_{\mathrm{i}}$ is the inertia constant in seconds, $\mathrm{T}_{\mathrm{mi}}$ is the mechanical input power in p.u., $\mathrm{E}_{\mathrm{qi}}^{\prime}$ is the q-axis internal transient electric potential of the $\mathrm{i}$-th generator in p.u., $\mathrm{E}_{\mathrm{fdi}}$ is the control signal in p.u., Uf is the excitation system control signal and $\mathrm{Ug}$ is the speed governor control signal and in the form of state equation each machine can be expressed by:

$$
\begin{aligned}
& \dot{\mathrm{X}}=\mathrm{F}(\mathrm{X})+\mathrm{G} \cdot \mathrm{U} \\
& \mathrm{Y}=\mathrm{H}(\mathrm{X})
\end{aligned}
$$

where

$$
\begin{aligned}
& \mathrm{X}=\left\lfloor\Delta \delta, \Delta \omega, \mathrm{E}_{\mathrm{q}}^{\prime}, \mathrm{E}_{\mathrm{fd}}, \mathrm{P}_{\mathrm{m}}\right\rfloor \\
& \mathrm{U}=\left[\mathrm{U}_{\mathrm{f}}, \mathrm{U}_{\mathrm{G}}\right] \\
& \mathrm{Y}=\left[\Delta \delta, \Delta \mathrm{V}_{\mathrm{t}}\right]=\left[\mathrm{h}_{1}(\mathrm{X}), \mathrm{h}_{2}(\mathrm{X})\right] \\
& \mathrm{G}=\left[\mathrm{g}_{1}, \mathrm{~g}_{2}\right] \\
& \mathrm{g}_{1}=\left[\begin{array}{lllll}
0 & 0 & 0 & \frac{\mathrm{K}_{\mathrm{A}}}{\mathrm{T}_{\mathrm{A}}} & 0
\end{array}\right]^{\mathrm{T}} \\
& g_{2}=\left[\begin{array}{lllll}
0 & 0 & 0 & 0 & \frac{K_{G}}{T_{G}}
\end{array}\right]^{T}
\end{aligned}
$$

By the above assumptions for each machine the nonlinear fuzzy controller discussed and SA optimization algorithm below is designed to control and stabilize the system in face of a load disturbance occurred in system.

\section{THE FuZZY LOGIC CONTROLLER}

Fuzzy set theory and fuzzy logic establish the rules of a non-linear mapping. The use of fuzzy sets provides a basis for a systematic way for the application of uncertain and indefinite models. Fuzzy set is based on a logical system called fuzzy logic controller. It is much closer in spirit to human thinking and natural language than classical logical systems. Nowadays, fuzzy logic is used in almost all sectors of industry and science. One of them is the LFC problem. Because of the complexity and multivariable conditions of the power system, conventional control methods may not give satisfactory solutions. On the other hand, robustness and reliability make the FLC useful in solving a wide range of control problems. Compared to conventional control techniques, FLC offers three important benefits. First, developing a FLC is cheaper than developing a model based or other controller with comparable performance. Second, FLC is more robust than PID controllers because they can cover a much wider range of operating conditions than PID controllers. Third, FLC is customizable, because it is easier to understand and modify their rules, which not only mimic a human operator's strategies, but also are expressed in linguistic terms used in natural language.

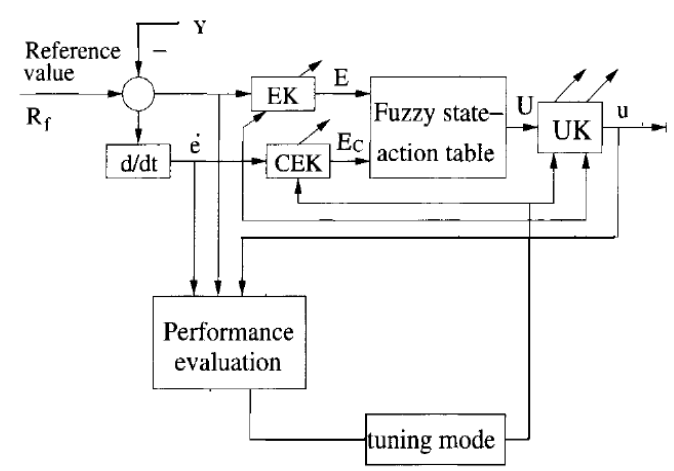

Fig. 2. The block diagram of fuzzy self tuning control system

But a major limitation in the simple fuzzy control system (SFCS) is the lack of a systematic methodology for developing fuzzy rules. A set of fuzzy rules often needs to be manually adjusted by trial-and-error before it reaches the desired level of performance. This limitation of SFCS is related to a more general problem in process control, namely, the difficulty in predicting changes in the operating conditions of a plant and then adjusting for them. Hence, it is desirable to develop a fuzzy self-tuning controller that can improve its performance based on its experience, and to adapt its response in relation to variations in the process dynamics, Fig 2 demonstrate according such system. To implement the function above, the designer of a fuzzy selftuning controller needs to deal with three things simultaneously: dynamic characteristics recognition of a plant, definition of performance index, and design a method for self-tuning of controller parameters, respectively. In this 
paper after the modeling of the system and definition of performance index, then in order to do the self tuning controller parameters, SA algorithm is done to move forward a big step from the SFCS and to demonstrate the benefits of this method.

\section{Simulated AnNeAling Optimization Algorithm}

In statistical mechanics, a physical process called annealing is often performed in order to relax the system to a state with minimum free energy. In the annealing process, a solid in a heat bath is heated up by increasing the temperature of the bath until the solid is melted into liquid, then the temperature is lowered slowly. In the liquid phase all particles of the solid arrange themselves randomly. In the ground state the particles are arranged in a highly structured lattice and the energy of the system is minimum. The ground state of the solid is obtained only if the maximum temperature is sufficiently high and the cooling is done sufficiently slowly. Based on the annealing process in the statistical mechanics, the SA was introduced for solving complicated combinatorial optimization. SA is effective in network reconfiguration problems for large-scale distribution systems, and its search capability becomes more significant as the system size increases. Moreover, the cost function with a smoothing strategy enables the SA to escape more easily from local minima and to reach rapidly to the vicinity of an optimal solution. The SA algorithm starts with the generation of a single random candidate solution, known as the current state. The objective function is evaluated with this initial candidate solution. The next solution is generated by a slight perturbation of the current state. Generation of the next state from the previous state is known as making the move. If the value of the objective function evaluated from this move is less than that of the current value, then the new move is accepted as the current solution. Even if the new move makes the value of the objective function greater than the current value, it is accepted with a certain probability.

The uphill move prevents SA from getting trapped into a local minimum. The above steps are repeated according to a well-defined annealing schedule till the control parameter known as the "temperature" reaches its end value. In Fig 2 , tuning mode adjusts the scaling factors (i.e., EK, CEK, and UK) by SA algorithm which makes the performance index optimum and satisfied. In general, the effectiveness of the tuning mode of fuzzy self tuning control system is better than that of a simple fuzzy controller, simulation result will approve this subject.

\section{IMPLEMENTATION AND RESULTS}

Simulations were performed using the conventional PI, Non-optimal fuzzy logic and the proposed optimal fuzzy logic controllers applied to a two-area interconnected power system as shown in Fig. 1 by applying a step load disturbance to both areas. The same system parameters, given in the Appendix, were used in all controllers for a comparison. The implementation worked with Matlab 7.9Simulink software and Fuzzy Logic Toolbox. In the optimization, the integral absolute error of the frequency deviation of the first area is selected as the performance index. Accordingly, the objective function $\mathrm{J}$ is set by

$$
J=\int_{0}^{\infty}|\Delta \mathrm{F} 1| \mathrm{dt}
$$

Before the implementation of the SA algorithm on the plant, the influence of scale factor changes on the plant is discussed here, first the influence of change in EK in Figure 2. From Figure 2, if EK changes, the value of the linguistic variable corresponding to the error in fuzzy controller will change too. Generally speaking, when EK increases, the value of the linguistic variable corresponding to the error will increase, and vice versa. Figure 3 depicts a comparison after EK is tuned when other parameters are fixed. The curves show the step responses of the plant. Then we conclude the influence to the system performance of EK as follows: smaller value of EK will lead to faster system response speed and the rising time would be small, Larger values of EK will lead to slower system response speed and the rising time would be large too, If EK is very large, this leads to very long settling time. The system may become oscillatory and even unstable.

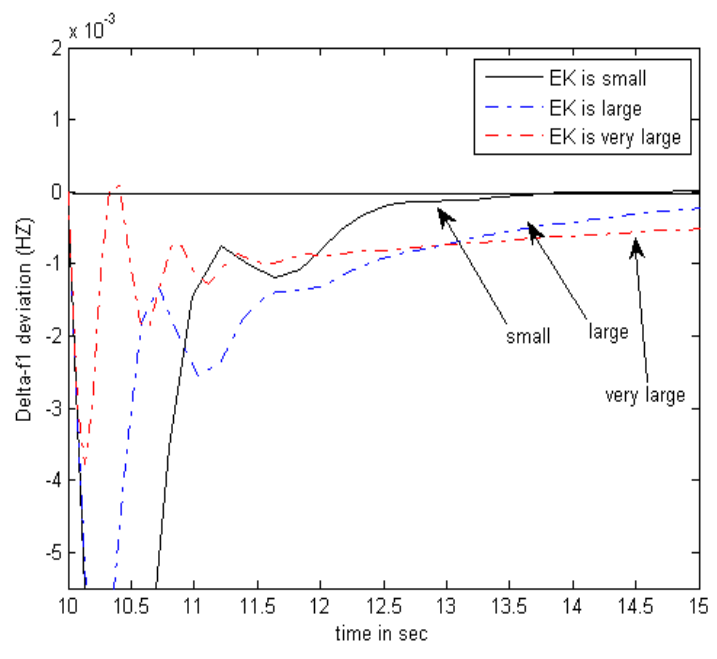

Fig. 3. Comparison of the influence of change in EK

The influence of the CEK is as follows : If CEK is very large, it leads to a long transient time, and even divergent system output. On the contrary, if CEK is very small, it makes the size of the overshoot, the degree of oscillation, and the size of the undershoot increase simultaneously. And the value of UK influences the system performance directly, If UK is very large, it leads to fluctuation and even divergence of system's output. If UK is very small, forward transmission gain of the system will be small, which reduces the response speed.

After tuning, the scaled factors by SA correspond to the optimal value The frequency deviations of the first area after a sudden load change are shown in Fig. 3 . The nonoptimal FLPI controller highly improves the system performance comparison to the conventional PI controller. Moreover, the optimal FLPI controller is significantly superior to the conventional PI controller. It gives a better performance than the non-optimal FLC. The settling time and overshoot are reduced considerably. In contrast, the values of the settling time, overshoot, and performance index in case of the optimal FLC are lower and rarely change. 


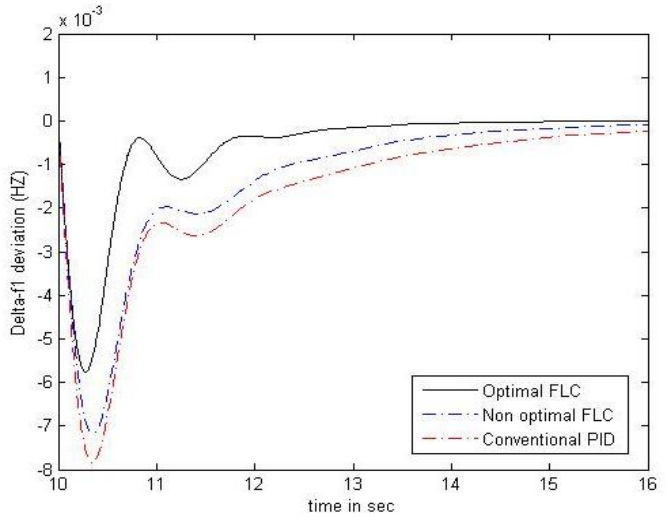

Fig. 4. The frequency deviations of the first area

This clarifies that the robustness of the optimal FLC against parameters variations is superior to that of the conventional PI and simple FLC. Finally, the frequency control effects of conventional PI, non-optimal FLC, and optimal FLC controllers are evaluated under different random step load variations that are applied to both areas as indicated in Fig. 5. The result of the frequency deviations of the first area is shown in Fig. 6.

The frequency deviations for the first area are improved considerably by the optimal FLC in comparison with the case of the conventional PI, non-optimal FLC.

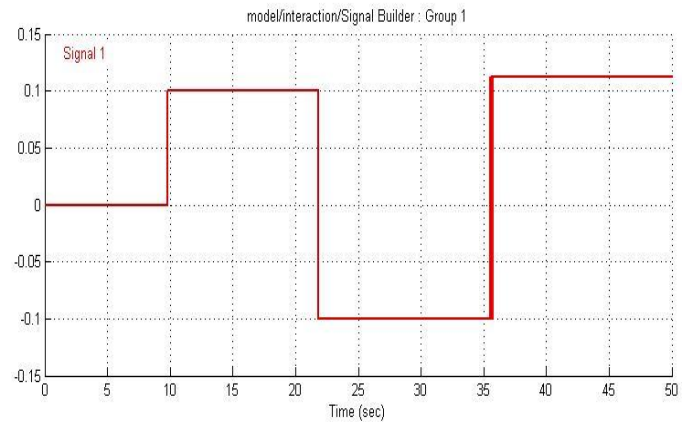

Fig. 5. The different random step load variations

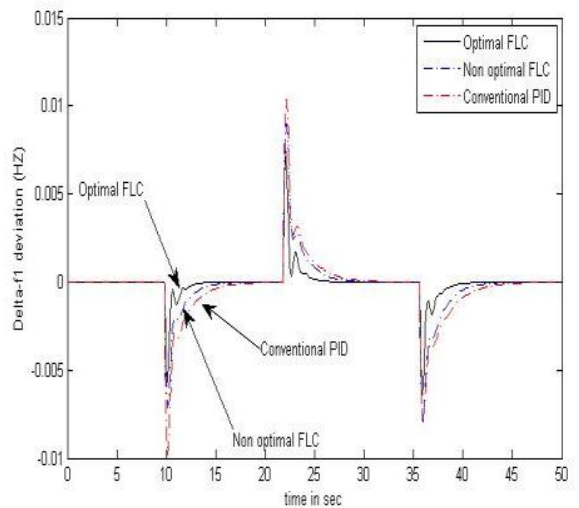

Fig. 6. The frequency deviations of the first area after different step load change

\section{CONCLUSION}

In this paper, a new approach, called the SA algorithm has been used for developing an optimal fuzzy logic controller for the LFC of a two-area interconnected power system. The proposed technique for designing a FLC helps us save time when compared to those from conventional trial and error design procedures. Another benefit of this approach is that it does not require experts for the design of the fuzzy logic controller. Finally, the simulation results indicate that the proposed FLC performs significantly better than other controllers in the areas of settling time, overshoot, and absolute error integral. Therefore, this proposed FLC is effective, efficient and robust over a wide range of operating conditions.

TABLE I: GENERATOR CONSTANTS

\begin{tabular}{|c|c|c|}
\hline & Generator G1 & Generator G2 \\
\hline $\mathrm{Xd}[\mathrm{p} . \mathrm{u}]$ & 1.569 & 1.651 \\
\hline $\mathrm{Xd}[\mathrm{p} . \mathrm{u}]$ & 0324 & 0.232 \\
\hline $\mathrm{Xd} "[\mathrm{p} . \mathrm{u}]$ & 0.249 & 0.171 \\
\hline $\mathrm{Xq}[\mathrm{p} . \mathrm{u}]$ & 1.548 & 1.590 \\
\hline $\mathrm{Xq}$ 'p.u] & 0.918 & 0.380 \\
\hline $\mathrm{Xq} "[\mathrm{p} . \mathrm{u}]$ & 0.248 & 0.171 \\
\hline $\mathrm{xl}[\mathrm{p} . \mathrm{u}]$ & 0.204 & 0.102 \\
\hline Td0's] & 5.140 & 5.900 \\
\hline Td0" [s] & 0.0437 & 0.033 \\
\hline $\mathrm{Tq} 0[\mathrm{~s}]$ & 0.500 & 0.535 \\
\hline Tq0" [s] & 0.070 & 0.078 \\
\hline $\mathrm{D}$ [p.u] & 2.000 & 2.000 \\
\hline$\omega 0=2 \pi \mathrm{t}[\mathrm{rad} / \mathrm{s}]$ & $120 \pi$ & $120 \pi$ \\
\hline $\mathrm{H}[\mathrm{MW}-\mathrm{Sec} / \mathrm{MVA}]$ & 50.00 & 9.00 \\
\hline Capacity[GVA] & 6000 & 2 \\
\hline
\end{tabular}

\section{REFERENCES}

[1] N. Jaleeli, L. S. VanSlyck, D. N. Ewart, L. H. Fink, and A. G Hoffmann, "Understanding automatic generation control," IEEE Trans. On Power Systems, vol. 7, no. 3, pp. 1106-1112, 1992.

[2] C. S. Chang and F. Weihui, "Area loadfrequency control using fuzzy gain scheduling of PI controllers," Electr. Power Syst. Res, vol. 42, pp. 145-152, 1997.

[3] J. Talaq and F. AL-Basri, "Adaptive fuzzy gain scheduling for load frequency control," IEEE Trans. on Power Systems, vol. 14, no. 1, pp. 145- 150, 1999.

[4] O. P. Malik, A. Kumar, and G. S. Hope, "A load frequency control algorithm based on a generalized approach," IEEE Trans. Power Systems, vol. 3, no. 2, pp. 375-382, 1988.

[5] N. Saha and S. P. Ghoshal, "State adaptive optimal generation control of energy systems," JIEEE, vol. 67, Part EL-2, 1986.

[6] A. Kumar, O. P. Malik, and G. S. Hope, "Variable structure-system control applied to AGC of an interconnected power system," IEE Proc. 132, Part C, no. 1, pp. 23-29, 1985.

[7] Z. M. A. Hamouz and Y. L. A. Magid, "Variable-structure loadfrequency controllers for multi area power systems," Int. J. Electr. Power Energ. Syst, vol. 15, no. 5, pp. 23-29, 1993.

[8] C. T. Pan and C. M. Liaw, "An adaptive control using fuzzy logic,' IEEE Trans. on Power Systems, vol. 4, no. 1, pp. 122-128, 1989.

[9] C. S. Indulkar and B. Raj, "Application of fuzzy controller to automatic generation control," Electric Mach. Power Systems, vol. 23, no. 2, pp. 209-220, 1995 .

[10] D. K. Chaturvedi, P. S. Satsangi, and P. K. Kalra, "Load frequency control: A generalized neural network approach," Electrical Power and Energy Systems, vol. 21, pp. 405-415, 1999.

[11] A. Hariri and O. P. Malik, "Fuzzy logic power system stabilizer based on genetically optimized adaptive network," Fuzzy Sets Systems, vol. 102, pp. 31-40, 1999.

[12] C. Ertugrul and I. Kocaarslan, "A fuzzy gain scheduling PI controller application for an interconnected electrical power system," Electr. Power Syst. Res, vol. 73, pp. 267-274, 2005.

[13] C. Ertugrul and I. Kocaarslan, "Load frequency control in two area power systems using fuzzy logic controller," Energy Conversion and Management, vol. 46, pp. 233-243, 2005

[14] M. E. E. Hawary, "Electric Power Applications of Fuzzy Systems,' IEEE Press, New York, 1998.

[15] F. Glover, "Tabu search part I," ORSA J. Comput, vol. 1, no. 3, pp 190-206, 1989

[16] F. Glover, "Tabu search part II," ORSA J. Comput, vol. 2, no. 1, pp. 4-32, 1990. 
[17] J. A. Bland and G. P. Dawson, "Tabu search and design optimization," Computer-Aided Design, vol. 23, no. 3, pp. 195-201, 1991.

[18] M. Denna, G. Mauri, and A. M. Zanaboni, "Learning fuzzy rules with tabu search-an application to control," IEEE Trans. on Fuzzy Systems, vol. 7, pp. 295-318, 1999.

[19] D. Karaboga, "Design of fuzzy logic controllers using tabu search algorithm," Fuzzy Information Processing Society, NAFIPS, 1996 Biennial Conference of the North American, pp. 489-491, 1996.

[20] C. C. Lee, "Fuzzy logic in control systems: Fuzzy logic controller, parts I-II.," IEEE Trans. Syst. Man Cyber, vol. 20, no. 2, pp. 404-418, 1990.

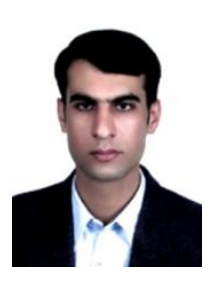

Mahmoud Najafi Applied Electronic Engineer by National university of Iran (2003) And Master of science In Electrical Control Engineering by National Azad University Of Iran (2010) .Has worked about 10 years at the Industrial Control and Instrument Department Of Iranian Offshore oil Company (IOOC). Has taken part and lead several projects to Design and develop instrumentation and control Systems for process industry. His current areas of interest include intelligent and digital control and IT and Communication systems. Now is ICT Manager of IOOC. 